\title{
Assessment of a New Ginsenoside Rh2 Nanoniosomal Formulation for Enhanced Antitumor Efficacy on Prostate Cancer: An in vitro Study
}

This article was published in the following Dove Press journal:

Drug Design, Development and Therapy

\begin{abstract}
Hadi Zare-Zardini (D) ${ }^{1-3}$
Ashraf Alemi iD ${ }^{4}$

Asghar Taheri-Kafrani ${ }^{5}$

Seyed Ahmad Hosseini ${ }^{6}$

Hossein Soltaninejad ${ }^{7,8}$

Amir Ali Hamidieh ${ }^{9}$

Mojtaba Haghi Karamallah (D) ${ }^{10}$

Majid Farrokhifar "I

Mohammad Farrokhifar ${ }^{12}$

'Hematology and Oncology Research Center, Shahid Sadoughi University of Medical Sciences, Yazd, Iran;

${ }^{2}$ Department of Sciences, Farhangian University, Isfahan, Iran; ${ }^{3}$ Medical

Nanotechnology \&Tissue Engineering

Research Center, Yazd Reproductive

Sciences Institute, Shahid Sadoughi

University of Medical Sciences, Yazd, Iran;

${ }^{4}$ Abadan Faculty of Medical Sciences,

Abadan, Iran; ${ }^{5}$ Department of

Biotechnology, Faculty of Advanced

Sciences and Technologies, University of

Isfahan, Isfahan, Iran; ${ }^{6}$ Nutrition and

Metabolic Diseases Research Center,

Ahvaz Jundishapur University of Medical

Sciences, Ahvaz, Iran; ${ }^{7}$ Tissue Bank \&

Research Center, Tehran University of

Medical Sciences, Tehran, Iran;

${ }^{8}$ Department of Nanobiotechnology,

Faculty of Biological Sciences, Tarbiat

Modares University, Tehran, Iran; ${ }^{9}$ Stem

Cell and Regenerative Medicine Institute,

Tehran University of Medical Sciences,

Tehran, Iran; ${ }^{10}$ Shoushtar Faculty of

Medical Sciences, Shoushtar, Iran;

"Department of Pediatrics, Sabzevar

University of Medical Sciences, Sabzevar, Iran; ${ }^{12}$ Kar Higher Education Institute of

Rafsanjan, Rafsanjan, Iran
\end{abstract}

Correspondence: Ashraf Alemi

Tel/Fax +986I53384008

Email alemi.ashraf@gmail.com
Introduction: Ginsenoside $\mathrm{Rh} 2$, purified from the Panax ginseng root, has been demonstrated to possess anticancer properties against various cancerous cells including colorectal, breast, skin, ovarian, prostate, and liver cancerous cells. However, the poor bioavailability, low stability on gastrointestinal systems, and fast plasma elimination limit further clinical applications of Ginsenoside Rh2 for cancer treatments. In this study, a novel formulation of niosomal Ginsenoside Rh2 was prepared using the thin film hydration technique.

Methods: The niosomal formulation contained Span 60 and cholesterol, and cationic lipid DOTAP was evaluated by determining particle size distribution, encapsulation efficiency, the polydispersity index (PDI), and surface morphology. The cytotoxic effects of free Ginsenoside Rh2 and Ginsenoside Rh2-loaded niosomes were determined using the MTT method in the PC3 prostate cancer cell line. For the investigation of the in vitro cellular uptake of Ginsenoside Rh2loaded niosome, two formulations were prepared: the Ginsenoside Rh2-loaded niosomal formula containing 5\% DOTAP and the Ginsenoside Rh2-loaded niosomal formula without DOTAP.

Results: The mean size, DPI, zeta potential, and encapsulation efficiency of the Ginsenoside Rh2-loaded nanoniosomal formulation containing DOTAP were 93.5 $\pm 2.1 \mathrm{~nm}, 0.203 \pm 0.01$, $+4.65 \pm 0.65$, and $98.32 \% \pm 2.4$, respectively. The niosomal vesicles were found to be round and have a smooth surface. The release profile of Ginsenoside $\mathrm{Rh} 2$ from niosome was biphasic. Furthermore, a two-fold reduction in the Ginsenoside Rh2 concentration was measured when Ginsenoside Rh2 was administered in a nanoniosomal form compared to free Ginsenoside Rh2 solutions in the PC3 prostate cancer cell line. After storage for 90 days, the encapsulation efficiency, vesicle size, PDI, and zeta potential of the optimized formulation did not significantly change compared to the freshly prepared samples. The cellular uptake experiments of the niosomal formulation demonstrated that by adding DOTAP to the niosomal formulation, the cellular uptake was enhanced.

Discussion: The enhanced cellular uptake and cytotoxic activity of the Ginsenoside Rh2 nanoniosomal formulation on the PC3 cell make it an attractive candidate for application as a nano-sized delivery vehicle to transfer Ginsenoside Rh2 to cancer cells.

Keywords: nanoniosomal, Ginsenoside Rh2, chemotherapy, PC3 prostate cancer cell line

\section{Introduction}

Prostate cancer is one of the most common cancers and the leading cause of cancer deaths among men worldwide. The best choices of treatment, radiation therapy and chemotherapy, are unsatisfactory due to their detrimental side effects on healthy cells and the problem of drug resistance. Searching for new agents for the treatment of cancer is the goal of numerous studies, and many studies are concentrated on plant-derived 
compounds that have curative potential. ${ }^{1-3}$ Ginsenoside $\mathrm{Rh} 2$ is one of the ginsenoside agents purified from the Panax ginseng root. ${ }^{4}$ This compound has different biological activities, especially an anticancer activity, against various cancerous cells (colorectal, breast, skin, ovarian, prostate, and liver cancer cells). ${ }^{5-7}$ The worst limitation for the application of Ginsenoside $\mathrm{Rh} 2$ is its low stability in gastrointestinal and bloodstream systems. Ginsenoside Rh2 has an extremely low oral bioavailability due to its high hydrophobic property. Moreover, it can be quickly eliminated from plasma. Ginsenoside Rh2 can be easily removed by enzymatic reactions in the body. ${ }^{8,9}$ Thus, enhancing the solubility and stability of Ginsenoside Rh2 is a major challenge for its application. For solving this problem, nanotechnology can be used as the best strategy to enhance the stability and efficiency of Ginsenoside Rh2. There are some studies on applications of nanostructures for this purpose. ${ }^{8,10}$ Accordingly, a lipid-based drug delivery system has been considered due to having fewer limitations than conventional formulations. Biocompatibility, biodegradability, efficiency, and large-scale production are the most important properties of lipid nanostructures. ${ }^{11,12}$ Previous studies used liposomes to enhance the efficiency of natural drugs such as Ginsenoside Rh2. ${ }^{8,13,14}$ There are no studies on the application of niosomes for this drug. Niosomes are non-ionic surfactant-based vesicles. Niosomes are formed mostly by non-ionic surfactants and cholesterol. Niosomes are a novel drug delivery system, in which the drug is encapsulated in a vesicle. ${ }^{15}$ Both hydrophilic and lipophilic drugs entrap in either the aqueous layer or the vesicular membrane. ${ }^{16}$ Niosomes are osmotically active and stable, increase the stability of the entrapped drug, are used for a variety of drugs, have controlled release, biodegradability, and non-immunogenic agents, and are biocompatible. ${ }^{17}$ To the best of our knowledge, no study has been performed on niosomes for delivery of Ginsenoside Rh2 to cancer cells, which was examined in the present study for the first time. Moreover, in the current study, the function of Ginsenoside Rh2 encapsulated in niosome was evaluated in comparison with free Ginsenoside $\mathrm{Rh} 2$ in terms of in vitro cellular uptake and cytotoxicity using the PC3 prostate cancer cell line.

\section{Materials and Methods}

\section{Preparation of Ginsenoside Rh2 Niosome}

A thin-film hydration method was employed for preparing Ginsenoside Rh2-loaded niosome. ${ }^{18-20}$ We dissolved Cholesterol (Sigma-Aldrich, USA), cationic lipid DOTAP (1,2-dioleoyl-3-trimethylammonium-propane, Sigma-
Aldrich, USA) (66.5:28.5: 5 molar ratios), and Span 60 (Sigma-Aldrich, USA) in chloroform as an oil phase (organic solution). Ginsenoside Rh2 (Sigma-Aldrich, USA) was then dissolved in one $\mathrm{mL}$ chloroform, and it was mixed with lipids/ surfactant. Cellular uptake was assessed by the addition of fluorescent label Dil (0.1 mol\%, Sigma, USA) to the lipid phase for lipid staining. The solvent (chloroform) was evaporated (organic phase removal) using a rotary evaporator (Heidolph, Germany) at $40^{\circ} \mathrm{C}$ as long as a thin-layered film was obtained. After preparation, we hydrated dry lipid film with phosphate-buffered saline ( $\mathrm{PBS}, \mathrm{pH}=7.4$ ) for 30 minutes at $50^{\circ} \mathrm{C}$ so that the niosomal suspensions are obtained. Once hydration was completed, sonication of vesicles was done for 20 minutes by a microtip probe sonicator for reducing the mean size of vesicles. When hydration was completed, a microtip probe sonicator was used for 45 minutes (15 s on - $10 \mathrm{~s}$ off, amplitude of 60 at 100 watts) for reducing the mean size of the vesicles. In the end, separation of free drugs (unloaded) from niosomal vesicles was done using dialysis tubing cellulose membrane (molecular weight cutoff $=12,000$, SigmaAldrich, USA) against PBS for one hour. ${ }^{21}$ Similarly, empty niosomes were developed, but Ginsenoside Rh2 was not added. The Ginsenoside Rh2 dosage was $0.2 \mathrm{mg} / \mathrm{mL}$. We prepared two formulations in order to investigate the in vitro cellular uptake in Ginsenoside Rh2-loaded niosome: a Ginsenoside Rh2-loaded niosomal formula that contained 5\% DOTAP and a Ginsenoside Rh2-loaded niosomal formula with no DOTAP. ${ }^{19}$

\section{Encapsulation Efficiency Analysis}

The entrapment effciency was determined using spectroscopic measurement. ${ }^{18,19}$ A UV spectrophotometer was used at 232 $\mathrm{nm}$ (Kmax) for estimating amounts of Ginsenoside $\mathrm{Rh} 2$ loaded into niosomes within the preparation. ${ }^{22}$ We mixed Ginsenoside Rh2-loaded niosome with isopropyl alcohol for lysing the membranes and allowing quick shedding of entrapped Ginsenoside $\mathrm{Rh} 2$ so that encapsulation effciency can be estimated. In the end, the following relation was used for determining encapsulation effciency:

Encapsulation effciency $(\%)=$
$\frac{\text { The amount of Ginsenoside Rh2 encapsulated within niosome }}{\text { Total amount of Ginsenoside Rh2 added }} \times 100$

\section{Determining Polydispersity Index, Size,} and Zeta Potential of Niosomal Vesicles

The polydispersity index (PDI) and particle size distribution of the obtained niosome were determined using the 
dynamic light scattering (DLS) method. To this end, a particle size analyzer was employed. The surface charge of the niosome was measured by the ZetaPALS zeta potential analyzer. A scanning electron microscope (SEM) was used for evaluating niosome surface morphology.

\section{In vitro Release Investigation of Ginsenoside Rh2 from Niosome}

One $\mathrm{mL}$ samples (formulations of Ginsenoside Rh2-loaded niosome) were prepared in dialysis tubes soaking in $10 \mathrm{~mL}$ PBS (at $\mathrm{pH}=7.4,37^{\circ} \mathrm{C}$ ) constantly stirring for 72 hours. Then, the profile of Ginsenoside Rh2 release from niosome was evaluated. Extraction of one $\mathrm{mL}$ of PBS directly surrounding the dialysis tube was done at pre-specified intervals, and UV/visible spectroscopy at $232 \mathrm{~nm}$ was used for reading the absorbance intensity. The release amount of Ginsenoside Rh2 was measured by the division of the amount of Ginsenoside Rh2 in the medium by its primary amount. $^{18,19,23}$

\section{Examination of Physical Stability}

The changes in zeta potential, particle size, PDI, and surplus amount of drug in the vesicle were examined at various periods for determining the physical stability of the optimal formulation of Ginsenoside Rh2-loaded niosome (formula with 5\% DOTAP) during storage. The niosome samples were sealed and kept in a glass vial for 90 days at $4^{\circ} \mathrm{C}$ under light protection. During 14 and 28 days, two and three-month intervals, stability analysis was done. $^{18,19,24,25}$

\section{Culture Conditions and Cell Lines}

We cultured human prostate cancer PC-3 cell (Iranian Biological Resource Center, Tehran, Iran) in DMEM with penicillin/streptomycin (Gibco, USA) and 10\% FBS (Fetal Bovine Serum, Gibco, USA) supplementation.

\section{Cytotoxicity Assays}

The MTT test was conducted for determining cell viability and comparing the inhibitory impact of free Ginsenoside Rh2 and Ginsenoside Rh2-loaded niosome on cell proliferation in test cells. ${ }^{26-28} 96$-well plates with $200 \mu \mathrm{L}$ of DMEM were used for seeding PC-3 cells, with 10\% FBS supplementation. The cells were seeded for one day, so that cell attachment happens. Then, $200 \mu \mathrm{L}$ fresh medium with series dilutions of Ginsenoside Rh-loaded niosome and free Ginsenoside Rh2 solution replaced the cell medium. Following one-day incubation, we added $20 \mu \mathrm{L}$ of MTT solution ( $5 \mathrm{mg} / \mathrm{mL})$ to the wells, and incubation of cells was done for $4 \mathrm{~h}$. The wells' supernatant was eliminated, followed by the addition of $180 \mu \mathrm{L}$ of DMSO to each well. An EPOCH Microplate Spectrophotometer at $570 \mathrm{~nm}$ was used for recording absorbance of each well. The cytotoxicity of Ginsenoside $\mathrm{Rh} 2$ as a niosomal drug or single drug was given as an $\mathrm{IC}_{50}$ value, which the concentration of drug needed for cell growth inhibition by $50 \%$ relative to an untreated control cell without treatment with Ginsenoside Rh2 as a niosomal drug or single drug. GraphPad Prism was used for calculating $\mathrm{IC}_{50}$ values.

\section{Cellular Uptake of Ginsenoside Rh2- Loaded Nanoniosome}

We prepared two formulations for analyzing in vitro cellular uptake of Ginsenoside Rh2-loaded niosome: the Ginsenoside Rh-loaded niosomal formula without DOTAP and Ginsenoside Rh2-loaded niosomal formula with 5\% DOTAP. The in vitro cellular uptake of Ginsenoside Rh2-loaded niosome was analyzed on PC-3 cells in 6-well plates at a density of 150,000 cells/well. Cell incubation was done for one day for cell attachment. Then, the treatment of cells with two formulations of Ginsenoside Rh2-loaded niosomes was performed during incubation for $4 \mathrm{~h}$ at $37^{\circ} \mathrm{C}$. Following a four-hour incubation process, the cells were rinsed three times using cold PBS, and then they were fixed with 4\% paraformaldehyde solution (Sigma, USA). Later, the DAPI solution was used for cell staining and a fluorescence microscope (Olympus, Japan) was used for imaging. ${ }^{21,26,28}$

\section{Statistical Analysis}

The tests were performed three times, and the data were provided as mean $\pm \mathrm{SD}$. Student's $t$-test was conducted as a statistical analysis to compare two independent groups. Data were considered significant at $p$-value $<0.05$.

\section{Results}

\section{Characterization of Ginsenoside Rh2- Loaded Nanoniosome}

The zeta potential, mean size, PDI, and encapsulation efficiency of the formulation of Ginsenoside Rh2-loaded nanoniosomes with DOTAP were $+4.65 \pm 0.65,93.5 \pm 2.1 \mathrm{~nm}$, $0.203 \pm 0.01$, and $98.32 \% \pm 2.4$ (Figure 1A and B). The zeta potential, mean size, PDI, and encapsulation efficiency of the formulation of Ginsenoside Rh2-loaded nanoniosome 


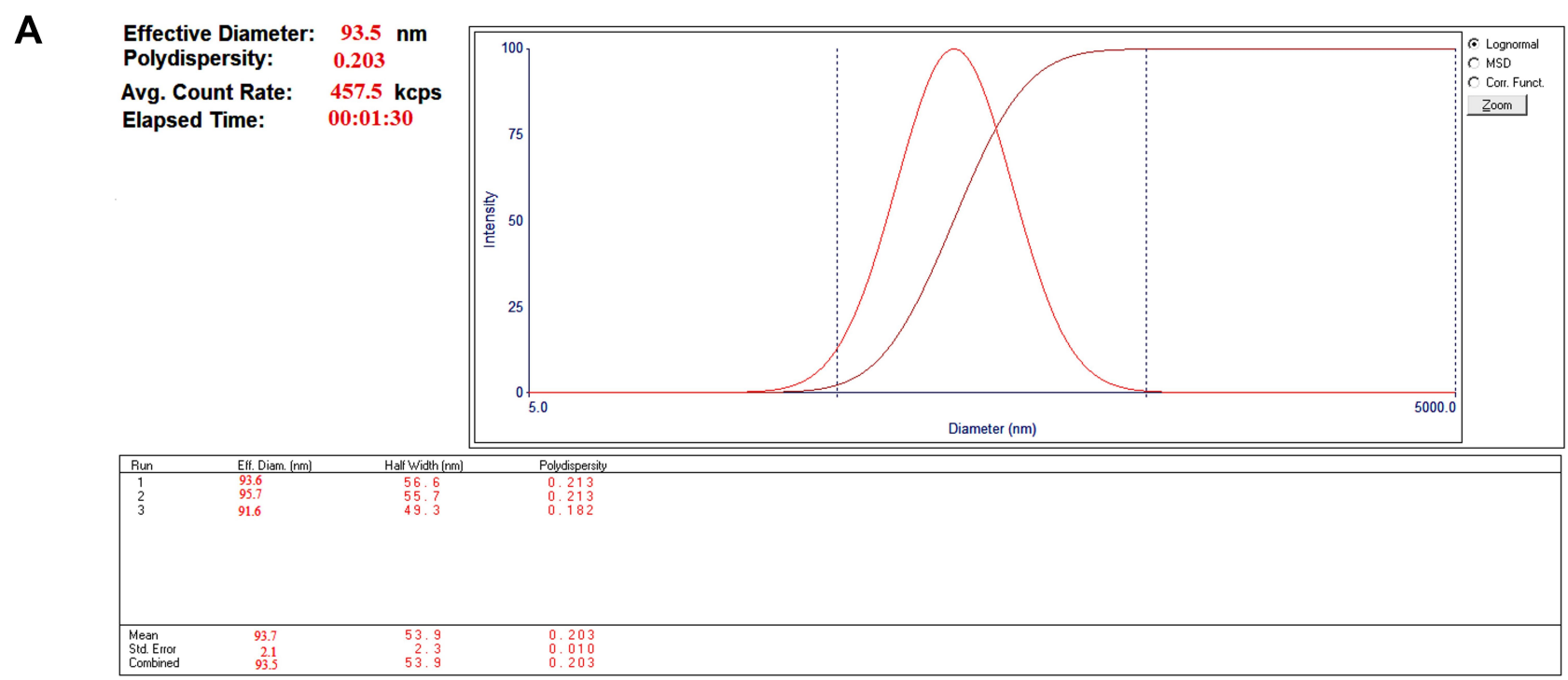

B

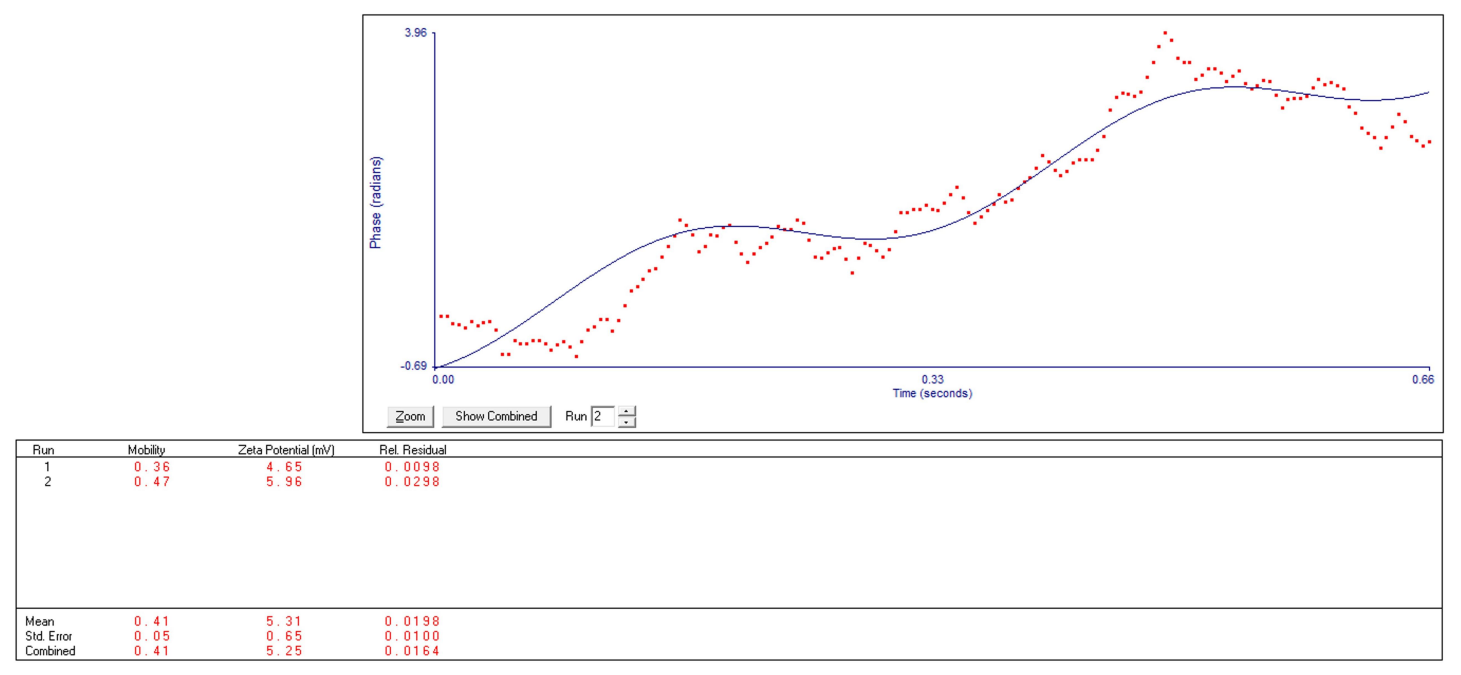

Figure I Physicochemical characteristics of formulation of Ginsenoside Rh2-loaded nanoniosome with DOTAP. (A) The size distribution and PDI formulation of Ginsenoside Rh2-loaded nanoniosome with DOTAP. (B) The zeta potential formulation of Ginsenoside Rh2-loaded nanoniosome with DOTAP.

without DOTAP were $-16.5 \pm 3.96,113.4 \pm 0.7 \mathrm{~nm}, 0.247$ \pm 0.011 , and $81.27 \% \pm 2.1$ (Figure $2 \mathrm{~A}$ and B). An SEM micrograph indicated that the formulations of Ginsenoside $\mathrm{Rh} 2$-loaded nanoniosome are in round shape with a smooth surface (Figure 3A and B).

\section{Investigation of in vitro Drug Release}

The dialysis method and dialysis tubing cellulose membrane were used for assessing in vitro release of the drug. Figure 4 indicates the profile of Ginsenoside $\mathrm{Rh} 2$ release from niosomal formulations at $37^{\circ} \mathrm{C}$. Following 72 hours, there were $33.75 \%$ and $42.09 \%$ release of loaded
Ginsenoside Rh2 from nanoniosome with DOTAP and Ginsenoside Rh2-loaded nanoniosome without DOTAP, respectively (Figure 4A and B). The Ginsenoside Rh2 had a biphasic release profile with a quick primary release, and then a slower release phase appeared.

\section{Examination of Physical Stability}

For determining the physical stability of the optimal formulation of Ginsenoside Rh2-loaded niosome (formulation with 5\% DOTAP) during storage, regarding encapsulation efficiency, PDI, vesicle size, and zeta potential were examined by storage at $4^{\circ} \mathrm{C}$. Following being 
A

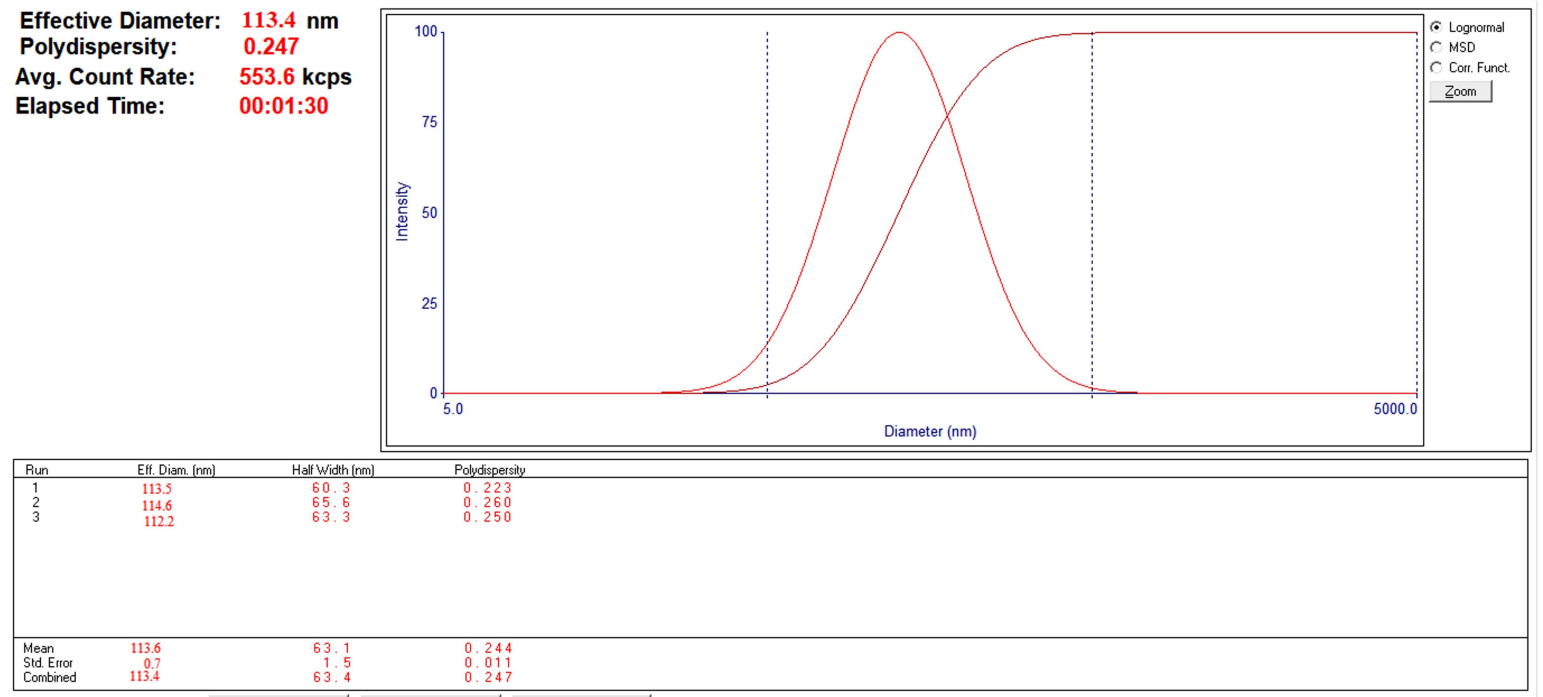

B

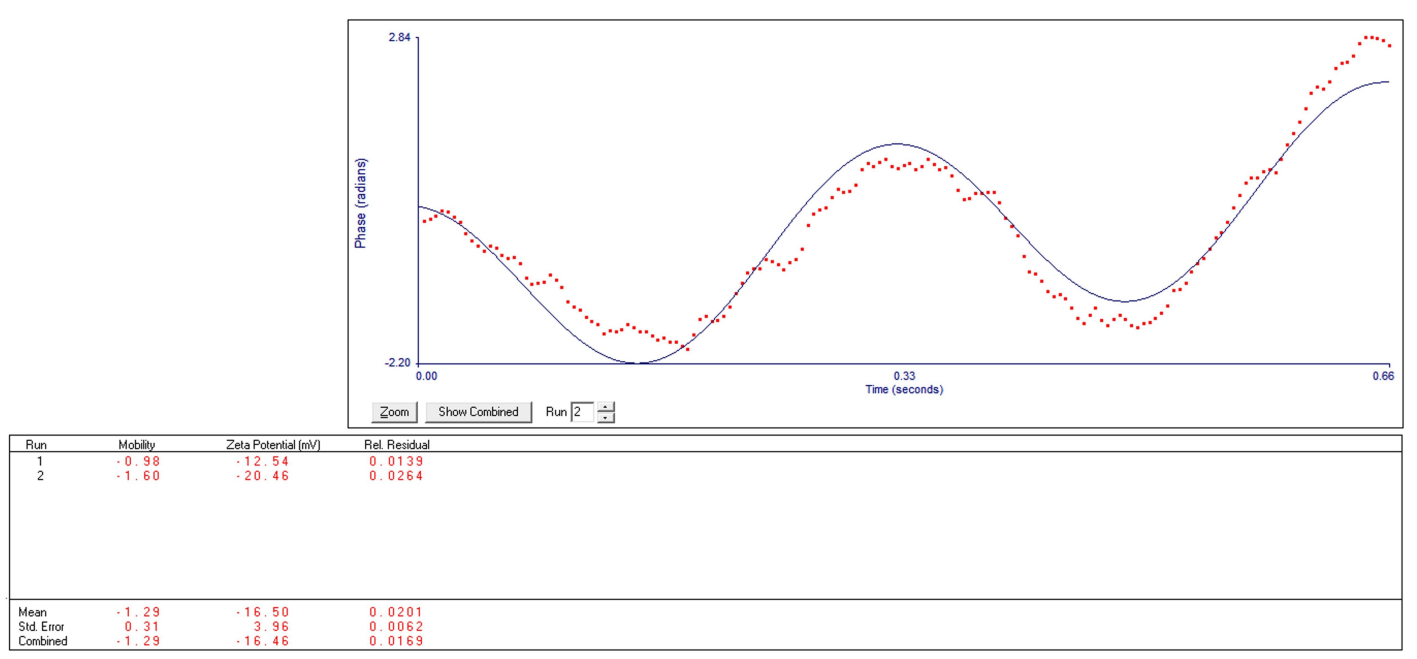

Figure 2 Physicochemical characteristics of formulation of Ginsenoside Rh2-loaded nanoniosome without DOTAP. (A) The size distribution and PDI formulation of Ginsenoside Rh2-loaded nanoniosome without DOTAP. (B) The zeta potential formulation of Ginsenoside Rh2-loaded nanoniosome without DOTAP.
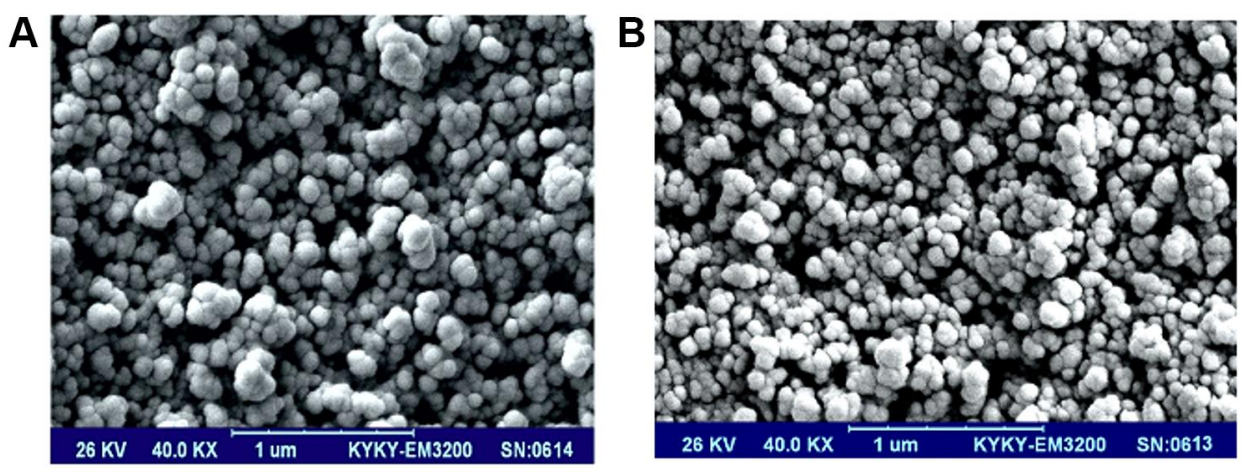

Figure 3 Morphological study of formulations of Ginsenoside Rh2-loaded nanoniosome. (A) Scanning electron microscopy of Ginsenoside Rh2-loaded nanoniosome with DOTAP. (B) Scanning electron microscopy of Ginsenoside Rh2-loaded nanoniosome without DOTAP. 

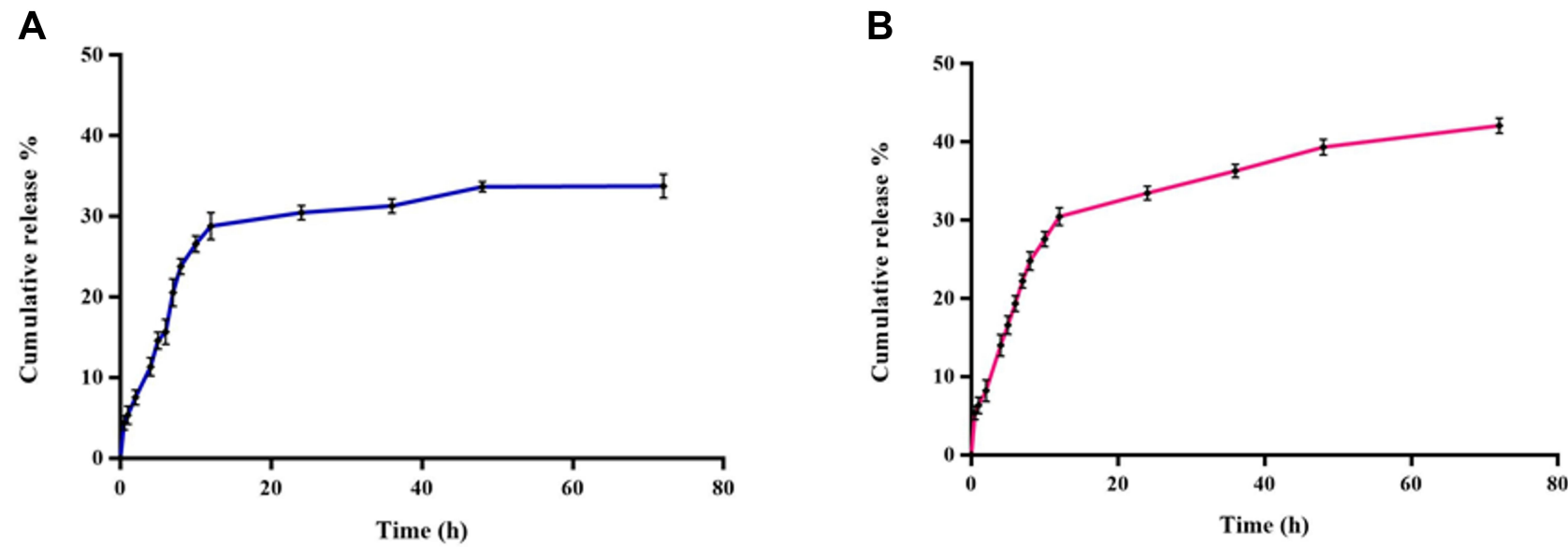

Figure 4 The in vitro release profile of Ginsenoside Rh2 from nanoniosome formulations. (A) The in vitro release profile of Ginsenoside Rh2 from nanoniosome formulation with DOTAP. (B) The in vitro release profile of Ginsenoside Rh2 from nanoniosome formulation without DOTAP.

stored for 90 days, a significant change was not observed in the PDI, vesicle size, encapsulation efficiency, and zeta potential of the optimized formula compared to the samples that were freshly generated ( $p$-value $>0.05)$. Thus, the stability of the Ginsenoside Rh2-loaded niosome formula with 5\% DOTAP was confirmed.

\section{Cytotoxicity Assays}

Dose-response tests were conducted for determining the inhibitory impact of Ginsenoside Rh2-loaded nanoniosome and drug-freeform on PC-3 cells. Figure 5 indicates that treating with drug niosomal form and drug-free form increased inhibition of PC-3 cells in a dose-dependent model. The $\mathrm{IC}_{50}$ values of drug-free form and drug niosomal form were $62.66 \pm 0.84$ and $31.24 \pm 0.75 \mu \mathrm{g} /$ $\mathrm{mL}$ against $\mathrm{PC}-3$ cells (Figure $5 \mathrm{~A}$ and $\mathrm{B}$ ). Figure 5B depicts that the niosomal formulation of Ginsenoside $\mathrm{Rh} 2$ had higher efficiency to deliver the Ginsenoside $\mathrm{Rh} 2$ to PC-3 cells. After administration of Ginsenoside Rh2, a two-fold decrease in the concentration of Ginsenoside Rh2 was found in the niosomal formula in comparison with the drug-free form.

\section{Cellular Uptake of Ginsenoside Rh2- Loaded Nanoniosome}

The cellular uptake behavior of Ginsenoside Rh2-loaded nanoniosome in PC-3 cells is indicated in Figure 6.
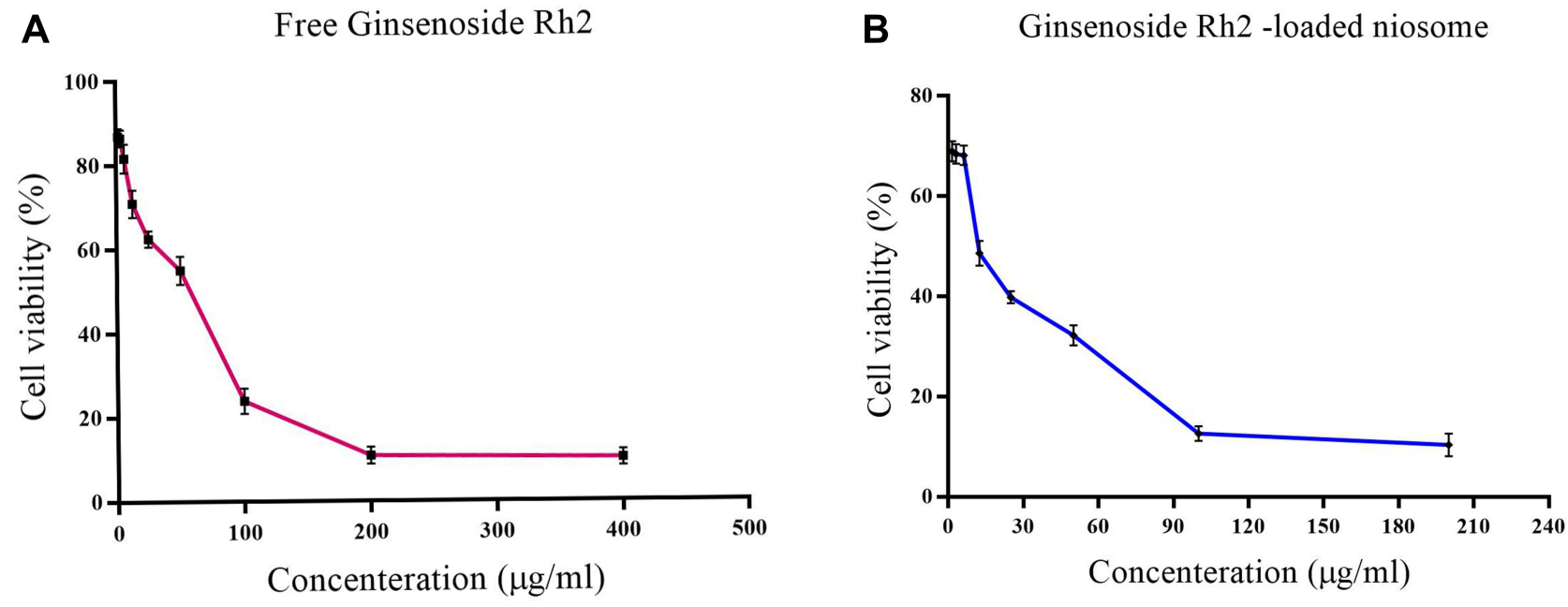

Figure 5 Cell viability assay on PC-3 cell line by Ginsenoside Rh2. (A) Cell viability on PC-3 cell after 48 hours of treatment with various concentrations of free Ginsenoside Rh2. (B) Cell viability on PC-3 cell after 48 hours of treatment with various concentrations of the optimal formulation of Ginsenoside Rh2-loaded nanoniosome. 


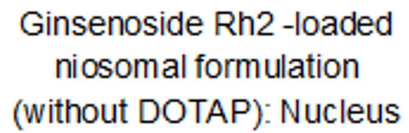

A

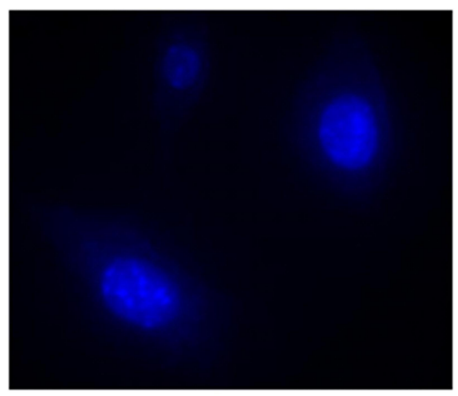

Ginsenoside Rh2 -loaded niosomal formulation (with DOTAP): Nucleus

B

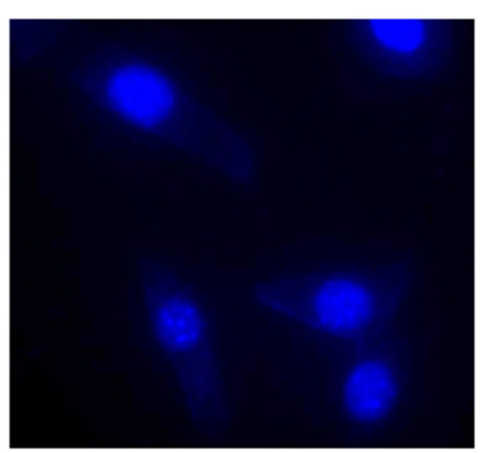

Ginsenoside Rh2 loaded

niosomal formulation

(without DOTAP)

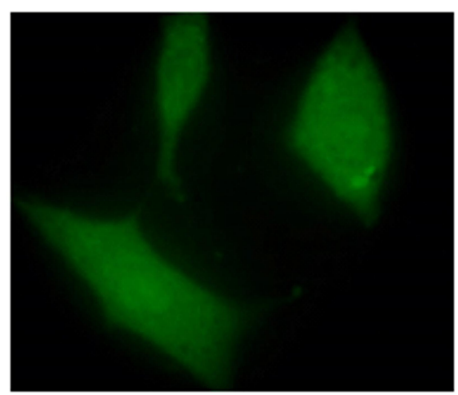

Ginsenoside Rh2 -loaded niosomal formulation (with DOTAP)

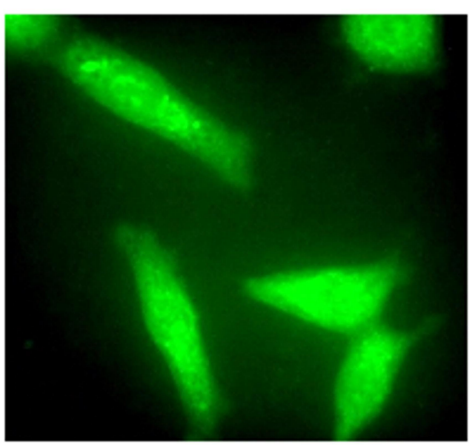

Ginsenoside Rh2 -loaded

niosomal formulation

(without DOTAP): Merged

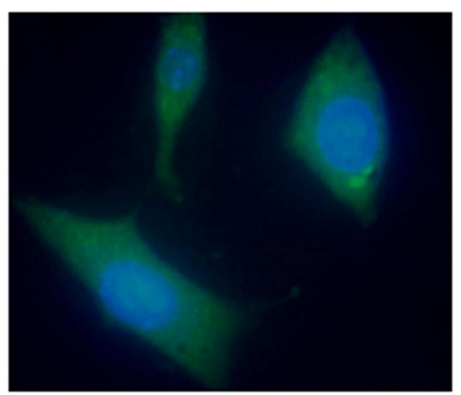

Ginsenoside Rh2 -loaded niosomal formulation (with DOTAP): Merged

Figure 6 Cellular uptake of Ginsenoside Rh2-loaded niosome on PC-3 line. (A) Ginsenoside Rh2-loaded niosomal formulation (without DOTAP). (B) Ginsenoside Rh2loaded niosomal formulation (with DOTAP).

Figure 6B shows that the PC-3 cells with Ginsenoside Rh2loaded niosomal treatment containing 5\% DOTAP had more green and cyan (blue-green) color intensity in comparison with PC-3 cells treated with Ginsenoside Rh2-loaded niosome formula without DOTAP (Figure 6A). Cellular uptake was improved with the addition of DOTAP to niosomal formulation. In addition, the behavior of Ginsenoside Rh2-loaded nanoniosome cellular uptake presented a higher distribution of Ginsenoside $\mathrm{Rh} 2$ in the nuclear area in comparison with the cytoplasm.

\section{Discussion}

In this study, a novel nanoniosomal formulation was synthesized to encapsulate Ginsenoside Rh2. The niosomal vesicular systems were prepared from the nonionic surfactant Span 60, as a commercial surfactant. The encapsulation efficiency of Ginsenoside Rh2-loaded nanoniosomal formulation containing DOTAP was $98.32 \%$. RationalThis high encapsulation efficiency may be speculated to be due mainly to the structural affinity between Ginsenoside Rh2 (as a hydrophobic agent) and the nonpolar stearate moiety (hydrophobic $\mathrm{C}-\mathrm{H}$ chain) of Span $60 .{ }^{23}$ Several other studies have attempted to determine a new formulation to obtain effective encapsulation for Ginsenoside Rh2. Chen et al synthesized novel double $\mathrm{pH}$-sensitive mixed micelles to fabricate multicore niosomes for drug delivery. The double $\mathrm{pH}$-sensitive mixed micelles (PMM) were prepared with different $\mathrm{pH}$-sensitive polymers, mPEG2000-Hz-CHEMS and mPEG2000-IS (2:1 w/w). Ginsenoside Rh2-loaded DPMM was mixed with Pluronic F-68 in the aqueous medium, and multicore niosomes were fabricated. The size of multicore niosomes 
was around 100-200 $\mathrm{nm}$ with the encapsulation efficiency around $93 \%{ }^{29}$ The cumulative release profile of Ginsenoside $\mathrm{Rh} 2$ from the nanoniosomal formulation was apparently biphasic with an initial rapid release period extended up to almost 12 hours followed by a slower release phase. The initial fast rate in the release occurring at the beginning phase can be regulated by the combination of diffuse mechanisms in the outside layers of niosomes and the emancipation of drugs located on the outer surface. In such situations, it was expected to transfer drug agents from the successional inner layer to the adjacent outer layers, and then, to the surface of the vesicle with delay. ${ }^{18,23,30}$ After storage for 90 days, the optimum formulation of Ginsenoside Rh2-loaded niosome (containing 5\% DOTAP) demonstrated no significant changes when compared to the freshly prepared samples in terms of encapsulation efficiency, vesicle size, polydispersity index (PDI), and zeta potential. This implies that the optimum formulation of Ginsenoside Rh2-loaded niosome could minimize problems associated with niosome instability, including aggregation, fusion, and drug leakage. ${ }^{18,19,24,25}$ Ginsenoside Rh2-loaded nanoniosome was synthesized and evaluated in terms of anticancer activities. The results indicated that treatment with the drug-free form and the drug niosomal form resulted in the growth inhibition of PC-3 cells in a dose-dependent pattern. Similar studies acquired identical data. ${ }^{31-33}$ Ginsenosides, especially Ginsenoside Rh2, are attractive candidates for anticancer drug development as they are relatively non-toxic to normal cells. Due to rapid proliferation, cancerous cells lose appropriate apoptotic control. Ginsenosides induce apoptosis in cancerous cells using the mitochondrial-dependent intrinsic apoptotic pathway and the death receptor-dependent extrinsic pathway. ${ }^{34-36}$ Thus, it is concluded that Ginsenoside $\mathrm{Rh} 2$ can lead to apoptosis induction in cancerous cells. Based on the data, a two-fold reduction was measured in the Ginsenoside $\mathrm{Rh} 2$ concentration, when Ginsenoside Rh2 was administered in the nanoniosomal form compared to free Ginsenoside Rh2 in PC-3 cells. Thus, the encapsulation of Ginsenoside Rh2 in the nanoniosomal form can lead to the reduction of the essential drug, but with similar effects as higher concentrations of free drug. The status of Ginsenoside $\mathrm{Rh} 2$ can reduce the cost of the therapeutic index of the used drug. The main challenge for the application of Ginsenoside Rh2 as a drug is its low stability in blood and GI systems. Encapsulation in delivery systems such as niosome can solve this challenge. Xin et al prepared a cocktail of betulinic acid, parthenolide, honokiol, and Ginsenoside $\mathrm{Rh} 2$ in liposome systems for lung cancer treatment by direct hydration of a lipid film. The average hydrodynamic size of the cocktail liposome system was $115.7 \mathrm{~nm}$ with a zeta potential of $-18.31 \mathrm{mV}$. The EE was $88.2 \%, 90.3 \%$, $89.5 \%$, and $91.4 \%$ for Ginsenoside Rh2, parthenolide, betulinic acid, and honokiol, respectively, and the total loading efficiency was $15.3 \%$. In vitro studies indicated that the combination of betulinic acid, parthenolide, honokiol, and Ginsenoside Rh2 exhibited a synergistic action. An increased effect was observed when these four natural products were loaded into liposome systems. ${ }^{37}$

The cellular uptake behavior of Ginsenoside $\mathrm{Rh} 2$ loaded nanoniosome in PC-3 cells was investigated by adding fluorescent label Dil to the lipid phase. The Ginsenoside Rh2-loaded niosomal formula containing cationic lipid DOTAP demonstrated greater green and cyan color intensity compared to the Ginsenoside Rh2loaded niosome formula without DOTAP. By adding DOTAP to niosomal formulation, cellular uptake was enhanced. On the other hand, the Ginsenoside Rh2loaded nanoniosomal cellular uptake behavior showed more Ginsenoside Rh2 distribution in the nuclear region compared to the cytoplasm. The large molecular size of Ginsenoside Rh2 leads to low membrane permeability. ${ }^{38,39}$ Due to its adsorption, endocytosis, and fusion, niosomes can penetrate into cells with high efficiency. ${ }^{18,19,40,41}$ Therefore, the cationic niosomal form of Ginsenoside Rh2 can enter cells with a higher quality compared to the niosomal form of Ginsenoside Rh2. Similar studies (eg, Han et al) showed the enhanced membrane permeability of ginsenosides with microemulsions. ${ }^{42}$

$\mathrm{Xu}$ et al optimized and prepared three types of Rh2loaded liposomes, including Rh2-loaded normal liposome (Rh2-LP), Rh2-loaded cationic liposome (Rh2-CLP), and $\mathrm{Rh}$ 2-loaded methoxy poly(ethylene glycol)-poly(lactide) (mPEG-PLA) liposome (Rh2-PLP), with the mean particle size of 80-125 nm. Rh2-LP presented better tumor accumulation of the fluorescentcyanine dye (DiR) in HepG2-xenografted nude mice than CLP (1.3-fold) or LP (1.6-fold) and prolonged the resident time of DiR in tumor and organs (more than $24 \mathrm{~h}$ ). The in vivo anticancer efficacy assessments indicate that $\mathrm{Rh} 2$-PLP presents the highest activity on suppressing tumor growth in HepG2-xenografted mice, compared to Rh2-LP and Rh2-CLP, without any significant toxicity. 


\section{Conclusions}

In conclusion, this study suggested a novel formulation of cationic niosomal Ginsenoside $\mathrm{Rh} 2$ to deliver drugs to the PC3 prostate cancer cell line. Ginsenoside Rh2 loading up to $93.5 \%$ was incorporated in the nanoniosomal bilayer with the formulation of Span 60, cholesterol, and DOTAP. The drug's release profile showed a burst release followed by a sustained drug release for Ginsenoside Rh2. After storage for 90 days, the optimum formulation of Ginsenoside Rh2-loaded niosome (containing 5\% DOTAP) demonstrated no significant changes when compared to freshly prepared samples in terms of encapsulation efficiency, vesicle size, PDI, and zeta potential. The Ginsenoside Rh2-loaded niosomal formula containing cationic lipid DOTAP demonstrated greater green and cyan color intensity compared to the Ginsenoside Rh2loaded niosome formula without DOTAP. By adding DOTAP to niosomal formulation, cellular uptake was enhanced. The enhanced cellular uptake and cytotoxic activity (two-fold) of Ginsenoside $\mathrm{Rh} 2$ nanoniosomal formulation with PC3 cells make it an attractive candidate for application as a nano-sized delivery vehicle to transfer Ginsenoside Rh2 to cancer cells.

\section{Disclosure}

The authors report no conflicts of interest in this work.

\section{References}

1. Azandeh SS, Abbaspour M, Khodadadi A, Khorsandi L, Orazizadeh M, Heidari-Moghadam A. Anticancer activity of curcumin-loaded PLGA nanoparticles on PC3 prostate cancer cells. Iran J Pharm Res. 2017;16(3):868-879.

2. Gonzalgo ML, Isaacs WB. Molecular pathways to prostate cancer. $J$ Urol. 2003;170(6,Part 1):2444-2452. doi:10.1097/01.ju.0000085381. 20139.b6

3. Richie JP. Anti-androgens and other hormonal therapies for prostate cancer. Urology. 1999;54(6,Supplement 1):15-18. doi:10.1016/S00904295(99)00449-5

4. Zhuang J, Yin J, Xu C, Mu Y, Lv S. 20(S)-Ginsenoside Rh2 induce the apoptosis and autophagy in U937 and K562 cells. Nutrients. 2018;10 (3):328. doi:10.3390/nu10030328

5. Wang M, Yan S-J, Zhang H-T, et al. Ginsenoside Rh2 enhances the antitumor immunological response of a melanoma mice model. Oncol Lett. 2017;13(2):681-685. doi:10.3892/ol.2016.5490

6. Song BK, Kim KM, Choi KD, Im WT. Production of the rare ginsenoside Rh2-MIX (20(S)-Rh2, 20(R)-Rh2, Rk2, and Rh3) by enzymatic conversion combined with acid treatment and evaluation of its anticancer activity. J Microbiol Biotechnol. 2017;27(7):1233-1241. doi:10.4014/jmb.1701.01077

7. Lee H, Lee S, Jeong D, Kim SJ. Ginsenoside Rh2 epigenetically regulates cell-mediated immune pathway to inhibit proliferation of MCF-7 breast cancer cells. J Ginseng Res. 2018;42(4):455-462. doi:10.1016/j.jgr.2017.05.003
8. Xu L, Yu H, Yin S, Zhang R, Zhou Y, Li J. Liposome-based delivery systems for ginsenoside Rh2: in vitro and in vivo comparisons. $J$ Nanopart Res. 2015;17(10):415. doi:10.1007/s11051-015-3214-Z

9. Lila ASA, Ishida T, Kiwada H. Targeting anticancer drugs to tumor vasculature using cationic liposomes. Pharm Res. 2010;27(7):11711183. doi:10.1007/s11095-010-0110-1

10. Zare-Zardini H, Taheri-Kafrani A, Amiri A, Bordbar A-K. New generation of drug delivery systems based on ginsenoside Rh2-, lysine- and arginine-treated highly porous graphene for improving anticancer activity. Sci Rep. 2018;8(1):586. doi:10.1038/s41598-01718938-y

11. Shrestha H, Bala R, Arora S. Lipid-based drug delivery systems. $J$ Pharm (Cairo). 2014;2014:801820.

12. Rahman MA, Harwansh R, Mirza MA, Hussain S, Hussain A. Oral lipid based drug delivery system (LBDDS): formulation, characterization and application: a review. Curr Drug Deliv. 2011;8(4):330345. doi:10.2174/156720111795767906

13. Hong C, Wang D, Liang J, et al. Novel ginsenoside-based multifunctional liposomal delivery system for combination therapy of gastric cancer. Theranostics. 2019;9(15):4437-4449. doi:10.7150/thno.34953

14. Kim H, Lee JH, Kim JE, et al. Micro-/nano-sized delivery systems of ginsenosides for improved systemic bioavailability. J Ginseng Res. 2018;42(3):361-369. doi:10.1016/j.jgr.2017.12.003

15. Kazi KM, Mandal AS, Biswas N, et al. Niosome: a future of targeted drug delivery systems. J Adv Pharm Technol Res. 2010;1(4):374380. doi:10.4103/0110-5558.76435

16. Sezgin-Bayindir Z, Antep MN, Yuksel N. Development and characterization of mixed niosomes for oral delivery using candesartan cilexetil as a model poorly water-soluble drug. AAPS PharmSciTech. 2014;16 (1):108-117. doi:10.1208/s12249-014-0213-9

17. Rajera R, Nagpal K, Singh SK, Mishra DN. Niosomes: a controlled and novel drug delivery system. Biol Pharm Bull. 2011;34(7):945953. doi:10.1248/bpb.34.945

18. Alemi A, Farrokhifar M, Zare-Zardini H, Haghi Karamallah MA. Comparison between the anticancer activities of free paclitaxel and paclitaxel-loaded niosome nanoparticles on human acute lymphoblastic leukemia cell line nalm-6. Iran J Ped Hematol Oncol. 2018;8 (3):153-160

19. Alemi A, Zavar Reza J, Haghiralsadat F, et al. Paclitaxel and curcumin coadministration in novel cationic PEGylated niosomal formulations exhibit enhanced synergistic antitumor efficacy. J Nanobiotechnology. 2018;16(1):28. doi:10.1186/s12951-018-0351-4

20. Uchegbu IF, Vyas SP. Non-ionic surfactant based vesicles (niosomes) in drug delivery. Int $J$ Pharm. 1998;172(1):33-70. doi:10.1016/ S0378-5173(98)00169-0

21. Lin YL, Liu YK, Tsai NM, et al. A Lipo-PEG-PEI complex for encapsulating curcumin that enhances its antitumor effects on curcumin-sensitive and curcumin-resistance cells. Nanomedicine. 2012;8 (3):318-327. doi:10.1016/j.nano.2011.06.011

22. Sharma V, Anandhakumar S, Sasidharan M. Self-degrading niosomes for encapsulation of hydrophilic and hydrophobic drugs: an efficient carrier for cancer multi-drug delivery. Mater Sci Eng C. 2015;56:393-400. doi:10.1016/j.msec.2015.06.049

23. Shaker DS, Shaker MA, Hanafy MS. Cellular uptake, cytotoxicity and in-vivo evaluation of tamoxifen citrate loaded niosomes. Int $J$ Pharm. 2015;493(1-2):285-294. doi:10.1016/j.ijpharm.2015.07.041

24. Ertekin ZC, Bayindir ZS, Yuksel N. Stability studies on piroxicam encapsulated niosomes. Curr Drug Deliv. 2015;12(2):192-199. doi:10.2174/1567201811666140723115852

25. Shilakari Asthana G, Sharma PK, Asthana A. In vitro and in vivo evaluation of niosomal formulation for controlled delivery of clarithromycin. Scientifica. 2016;2016:6492953. doi:10.1155/2016/6492953

26. Baek JS, Cho CW. A multifunctional lipid nanoparticle for co-delivery of paclitaxel and curcumin for targeted delivery and enhanced cytotoxicity in multidrug resistant breast cancer cells. Oncotarget. 2017;8(18):30369-30382. doi:10.18632/oncotarget.16153 
27. Lv S, Tang Z, Li M, et al. Co-delivery of doxorubicin and paclitaxel by PEG-polypeptide nanovehicle for the treatment of non-small cell lung cancer. Biomaterials. 2014;35(23):6118-6129. doi:10.1016/j. biomaterials.2014.04.034

28. Wang J, Wang F, Li F, et al. A multifunctional poly(curcumin) nanomedicine for dual-modal targeted delivery, intracellular responsive release, dual-drug treatment and imaging of multidrug resistant cancer cells †electronic supplementary information (ESI) available: the synthesis procedure of biotin-PEG-PCDA and the experimental results of MTT. J Mater Chem B Mater Biol Med. 2016;4(17):2954-2962.

29. Chen D, Yu H, Mu H, Li G, Shen Y. Novel multicore niosomes based on double $\mathrm{pH}$-sensitive mixed micelles for ginsenoside Rh2 delivery. Artif Cells Nanomed Biotechnol. 2014;42(3):205-209. doi:10.3109/ 21691401.2013.794358

30. Hong M, Zhu S, Jiang Y, Tang G, Pei Y. Efficient tumor targeting of hydroxycamptothecin loaded PEGylated niosomes modified with transferrin. J Control Release. 2009;133(2):96-102. doi:10.1016/j. jconrel.2008.09.005

31. Nag SA, Qin -J-J, Wang W, Wang M-H, Wang H, Zhang R. Ginsenosides as anticancer agents: in vitro and in vivo activities, structure-activity relationships, and molecular mechanisms of action. Front Pharmacol. 2012;3:25. doi:10.3389/fphar.2012.00025

32. Kim AD, Kang KA, Zhang R, et al. Ginseng saponin metabolite induces apoptosis in MCF-7 breast cancer cells through the modulation of AMP-activated protein kinase. Environ Toxicol Pharmacol. 2010;30(2):134-140. doi:10.1016/j.etap.2010.04.008

33. Li B, Zhao J, Wang C-Z, et al. Ginsenoside Rh2 induces apoptosis and paraptosis-like cell death in colorectal cancer cells through activation of p53. Cancer Lett. 2011;301(2):185-192. doi:10.1016/j. canlet.2010.11.015
34. Chen Y, Liu ZH, Xia J, et al. 20(S)-ginsenoside Rh2 inhibits the proliferation and induces the apoptosis of KG-1a cells through the Wnt/beta-catenin signaling pathway. Oncol Rep. 2016;36(1):137146. doi:10.3892/or.2016.4774

35. Sun M, Ye Y, Xiao L, Duan X, Zhang Y, Zhang H. Anticancer effects of ginsenoside Rg3. Int $J$ Mol Med. 2017;39(3):507-518. doi:10.3892/ijmm.2017.2857

36. Chen S, Wang Z, Huang Y, et al. Ginseng and anticancer drug combination to improve cancer chemotherapy: a critical review. Evid Based Complementary Altern Med. 2014;2014. doi:10.1155/2014/105186

37. Jin X, Yang Q, Cai N, Zhang Z. A cocktail of betulinic acid, parthenolide, honokiol and ginsenoside Rh2 in liposome systems for lung cancer treatment. Nanomedicine. 2020;15(1):41-54. doi:10. 2217/nnm-2018-0479

38. Li L, Chen X, Li D, Zhong D. Identification of 20(S)-protopanaxadiol metabolites in human liver microsomes and human hepatocytes. Drug Metab Dispos. 2011;39(3):472-483. doi:10.1124/dmd.110. 036723

39. Paek IB, Moon Y, Kim J, et al. Pharmacokinetics of a ginseng saponin metabolite compound $\mathrm{K}$ in rats. Biopharm Drug Dispos. 2006;27(1):39-45. doi:10.1002/bdd.481

40. Leung KW, Wong AS-T. Pharmacology of ginsenosides: a literature review. Chin Med. 2010;5(1):20. doi:10.1186/1749-8546-5-20

41. Xu Y-Q, Chen W-R, Tsosie JK, et al. Niosome encapsulation of curcumin: characterization and cytotoxic effect on ovarian cancer cells. J Nanomater. 2016;2016:9. doi:10.1155/2016/6365295

42. Han M, Fu S, Gao JQ, Fang XL. Evaluation of intestinal absorption of ginsenoside $\mathrm{Rg} 1$ incorporated in microemulison using parallel artificial membrane permeability assay. Biol Pharm Bull. 2009;32 (6):1069-1074. doi:10.1248/bpb.32.1069

\section{Publish your work in this journal}

Drug Design, Development and Therapy is an international, peerreviewed open-access journal that spans the spectrum of drug design and development through to clinical applications. Clinical outcomes, patient safety, and programs for the development and effective, safe, and sustained use of medicines are a feature of the journal, which has also been accepted for indexing on PubMed Central. The manuscript management system is completely online and includes a very quick and fair peer-review system, which is all easy to use. Visit http://www. dovepress.com/testimonials.php to read real quotes from published authors. 